\title{
Electrochemical preparation of nitrogen-doped graphene quantum dots and their size-dependent electrocatalytic activity for oxygen reduction
}

\author{
DHANRAJ B SHINDE ${ }^{1, *}$, VISHAL M DHAVALE ${ }^{1}$, SREEKUMAR KURUNGOT ${ }^{1}$ and \\ VIJAYAMOHANAN K PILLAI ${ }^{2}$ \\ ${ }^{1}$ Physical and Materials Chemistry Division, National Chemical Laboratory, Pune 411 008, India \\ ${ }^{2}$ Central Electrochemical Research Institute (CECRI), Karaikudi 630 006, India
}

MS received 17 September 2013; revised 2 February 2014

\begin{abstract}
Here we report a remarkable transformation of nitrogen-doped multiwalled carbon nanotubes (MWCNTs) to size selective nitrogen-doped graphene quantum dots (N-GQDs) by a two-step electrochemical method. The sizes of the N-GQDs strongly depend on the applied anodic potential, moreover increasing potential resulted in a smaller size of N-GQDs. These N-GQDs display many unusual size-dependant optoelectronic (blue emission) and electrocatalytic (oxygen reduction) properties. The presence of $\mathrm{N}$ dopants in the carbon framework not only causes faster unzipping of MWCNTs but also provides more low activation energy site for enhancing the electrocatalytic activity for technologically daunting reactions like oxygen reduction. The smaller size of N-GQDs has shown better performance as compared to the large N-GQDs. Interestingly, N-GQDs-3 (size $=\mathbf{2 . 5} \pm \mathbf{0 . 3}$ nm, onset potential $=0.75 \mathrm{~V})$ show a $30-\mathrm{mV}$ higher positive onset potential shift compared to that of $\mathrm{N}-\mathrm{GQDs}-2(\operatorname{size}=4.7 \pm 0.3 \mathrm{~nm}$, onset potential $=0.72 \mathrm{~V}$ ) and $70 \mathrm{mV}$ than that of $\mathrm{N}-\mathrm{GQDs}-1$ ( reduction reaction $(\mathrm{ORR})$ in a liquid phase. These result in the size-dependent electrocatalytic activity of N-GQDs for ORR as illustrated by the smaller sized N-GQDs $(2.5 \pm 0.3 \mathrm{~nm})$ undoubtedly promising metal-free electrocatalysts for fuel cell applications.
\end{abstract}

Keywords. N-GQDs; MWCNTs; fuel cells; unzipping; oxygen reduction reaction.

\section{Introduction}

The cathode reaction, namely oxygen reduction reaction (ORR), in proton exchange membrane fuel cells is more sluggish than the rate of hydrogen oxidation, ${ }^{1,2}$ and many researchers focused on improving the ORR activities using various platinum $(\mathrm{Pt})$-based and $\mathrm{Pt}$-free electrocatalysts. ${ }^{3,4}$ However, Pt is the noble metal and due to low abundance on earth it is very expensive to be commercialized. ${ }^{5}$ In recent studies, the carbon-based materials such as carbon nanotubes (CNTs), nanofibres and graphene have received attention as an alternative metal-free electrocatalyst to $\mathrm{Pt}^{6-8}$ Specifically, nitrogen N-doped carbon materials have been experimentally shown higher ORR activity, mainly following four-electron pathways. Moreover, the N-doped CNTs and graphene were also reported to have high activity for ORR even in the absence of metal atoms. On the other hand, the properties of nanocarbon sheets and graphene quantum dots (GQDs) have also been explored for many electrochemical and optoelectronic applications. ${ }^{9-12}$ Furthermore, GQDs are a few nanometre-sized carbon disks having many intrinsic characteristics, which exhibit remarkable photoluminescence (PL) in the visible region, unique electron transport features, as well as useful to various applications like light

*Author for correspondence (db.shinde@ ncl.res.in) emitting, organic photovoltaic devices, biological labelling etc. ${ }^{10}$ GQDs also possess low toxicity, high luminescence, chemical inertness and robust electrocatalytic activity for certain specific reactions like oxygen reduction, especially after doping with hetero-elements like nitrogen. ${ }^{13}$ Doping also helps to modify the electronic and chemical properties of GQDs, which could be contrasted with the other more common method of surface functionalization for modulating the behaviour. Nitrogen doping has been a powerful technique to modify graphitic carbonaceous materials for various applications like catalysis to nanoelectronics. ${ }^{14}$ Increase in nitrogen content with specific bonding configuration results in a debate on what configuration is optimal. Doping could also be used to modify the energy gap of GQDs and tune their electronic properties via bandgap engineering. Moreover, doping leads to disruption of the ideal $\mathrm{sp}^{2}$ hybridization of the carbon atoms in carbon allotropes, which also additionally introduce the n-type carriers in the system, thus locally inducing significant changes in their electronic properties as well as its chemical reactivity towards many catalytic reactions. ${ }^{9-12}$ However, a clear separation of contributions of intrinsic size dependence and doping is difficult in many systems even with a strong support from computational modelling and simulation groups. ${ }^{15} \mathrm{~N}$-GQDs have been studied theoretically, and electronic structural calculations suggest that nitrogen atoms located at their edge and near the 
edge might promote the electrocatalytic activity of N-GQDs towards the ORR. ${ }^{16,17}$ In spite of the huge potential of $\mathrm{N}-\mathrm{GQD}$, as an alternative to platinum, there are only very few experimental studies dealing with the synthesis and characterization of N-GQDs. For example, although ${ }^{18}$ reported the synthesis of N-GQDs from graphene oxide by using cyclic voltammetry $(\mathrm{CV})$ within the potential window $\pm 3 \mathrm{~V}$ in acetonitrile with tetra butyl ammonium per chlorate (TBAP) as supporting electrolyte, the exact source of nitrogen is unclear. Similarly, Li et $a l^{15}$ reported the behaviour of smaller-sized colloidal GQDs for ORR, but the electrocatalytic activity appears to be poor compared to Pt. Hence, there is an urgent need to clarify the performance of N-GQDs, especially with precise size control for ORR due to its enormous utility in fuel cells, electrolyzer and other electrochemical devices.

Herein, we report for the first time a unique synthetic strategy for N-GQDs using nitrogen-doped multi-walled carbon nanotubes (N-MWCNTs) as starting materials by means of a controlled application of interfacial electric field. Recently, we have reported an electrochemical transformation of MWCNTs to GQDs by a two-step process, ${ }^{17}$ where sustained oxidation of MWCNTs in propylene carbonate containing $\mathrm{LiClO}_{4}$ as supporting electrolyte at $1 \mathrm{~V}$ for $15 \mathrm{~h}$ at $90^{\circ} \mathrm{C}$ followed by reduction at $-1 \mathrm{~V}$ for $2 \mathrm{~h}$ each has yielded discrete spherical particles of pristine GQDs capable of unusual electron transfer behaviour. ${ }^{12,18,19}$ In comparison, here we describe the synthesis of N-GQDs of different sizes by applying positive bias like 2, 2.5 and $3 \mathrm{~V}$ (vs. QRE) to N-MWCNTs in acetonitrile containing $0.1 \mathrm{M}$ TBAP for $12 \mathrm{~h}$ each, followed by reduction in the same electrolyte at $-2 \mathrm{~V}$ for typically $4 \mathrm{~h}$ duration. The nitrogen content of N-GQDs remains essentially the same $(2.76 \%)$, although the yield systematically changed as a function of the oxidation potential and time. To the best of our knowledge, there is no prior report on the synthesis and characterization of N-GQDs using N-MWCNTs by an electrochemical approach. More importantly, this method represents a novel route for the preparation of size-selective N-GQDs, with different mechanism of unzipping compared with that of pristine MWCNTs. Also the electrocatalytic properties of different sized N-GQDs for ORR are evaluated to demonstrate their significant performance compared to other widely known electrocatalysts.

\section{Experimental}

\subsection{Materials}

Nitrogen-doped MWCNTs (3 wt\% N) purchased from Nanomaterials Inc., hydrochloric acid-HCl (36-37 wt\%), dimethy formamide (Aldrich) and highly purified water with a resistivity of greater than $18.0 \mathrm{M} \Omega \mathrm{cm}^{-1}$ were used in the preparation of aqueous solutions.

Nitrogen-doped MWCNTs (3 wt\% N) obtained from Nanomaterials Inc. were purified according to the following protocol. The sample $(50 \mathrm{mg})$ was heated for $12 \mathrm{~h}$ at $250^{\circ} \mathrm{C}$ in a furnace in argon atmosphere, further treated with $50 \mathrm{ml}$ of concentrated $\mathrm{HCl}$ thereby removing the metal catalysts in the form of their chlorides. The purified N-MWCNTs were dispersed in dimethyl formamide $\left(2 \mathrm{mg} \mathrm{ml}^{-1}\right)$ and drop casted $50 \mu \mathrm{l}$ on glassy carbon electrode (area $3 \mathrm{~mm}$ ) after polishing with alumina powders, rinsing thoroughly in acetone and after subsequent drying. Then GC electrode was kept under IR lamp for $1 \mathrm{~h}$ to remove solvents and to form a thin uniform layer. The electrochemical oxidation of $\mathrm{N}$ MWCNTs was carried out in a three-electrode system $(\mathrm{Pt}$ as counter electrode, $\mathrm{Pt}$ wire as reference electrode) with an Autolab PGSTAT30 (Eco Chemie) at a fixed positive bias like 2, 2.5 and $3 \mathrm{~V}$ (vs. QRE) in acetonitrile containing $0.1 \mathrm{M}$ TBAP for $12 \mathrm{~h}$ each followed by reduction in the same electrolyte at $-2 \mathrm{~V}$ for typically $4 \mathrm{~h}$ duration. The N-GQDs were purified by evaporating the acetonitrile from the solution and dissolving the remained materials in water, followed by dialysing the aqueous solution with a cellulose ester membrane bag (MD77(8000) at $60^{\circ}$ for 2 days to fully remove the supporting electrolyte of TBAP. The nitrogen content within $\mathrm{N}-\mathrm{GQD}$ remains essentially the same $2.76 \%$, although the yield systematically changed as a function of the oxidation potential and time.

\subsection{Characterizations}

Raman analysis of N-GQD and N-MWCNT samples was performed on an HR 800 Raman spectrometer (Jobin Yvon, Horiba, France) using 632.8 nm green laser (NRS $1500 \mathrm{~W}$ ). Transmission electron microscopy was carried out by a JEOL JEM 1200 EX operated at an accelerating voltage of $120 \mathrm{kV}$ with a resolution of not less than 3-4 nm. X-ray photoelectron spectroscopy (XPS) measurements were carried out on a VG MicroTech ESCA 3000 instrument at a pressure of $>1 \times 10^{-9}$ Torr (pass energy of $50 \mathrm{eV}$, electron takeoff angle $60^{\circ}$ and overall resolution $\sim 1 \mathrm{eV}$ ) using monochromatic $\mathrm{Mg}$ $\mathrm{K} \alpha$ (source, $h v=1253.6 \mathrm{eV}$ ). The core level spectra of the $\mathrm{C} 1 \mathrm{~s}$ and N1s orbitals were recorded with an overall instrumental resolution of $\sim 1 \mathrm{eV}$. The alignment of binding energy (BE) was carried out using $\mathrm{Au} 4 \mathrm{f} \mathrm{BE}$ of $84 \mathrm{eV}$ as the reference. X-ray flux (power $70 \mathrm{~W}$ ) was kept deliberately low to reduce the beam-induced damage. Spectra were fitted using a combined polynomial and Shirley type background function.

Steady-state fluorescence emission and variable temperature measurements were performed using a Fluoro log HORIBA JOBIN YVON on fluorescence spectrophotometer. The emission as well as excitation slit width was maintained at $1 \mathrm{~nm}$ throughout the experiments and the data were obtained in ' $\mathrm{S}_{\mathrm{c}} / \mathrm{R}_{1}$ ' mode (to account for the variations in lamp intensity).

\section{Results and discussion}

Figure 1 displays a comparison of the transmission electron microscopy (TEM) images of as prepared monodispersed N-GQDs with average sizes $2.5 \pm 0.3,4.7 \pm 0.3$ and 


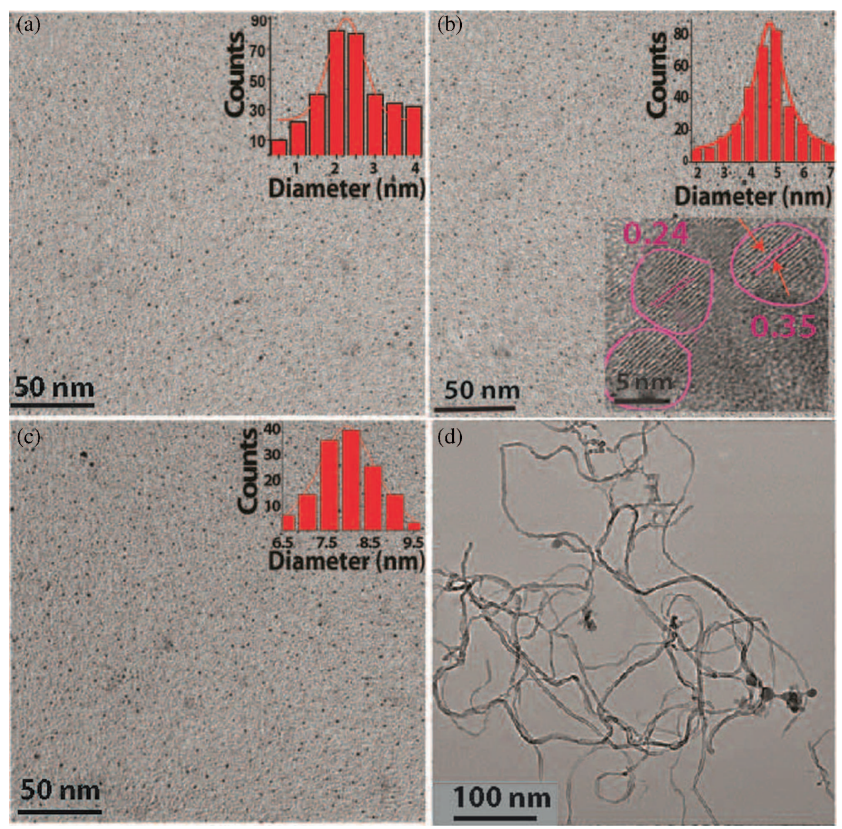

Figure 1. TEM images of 'as prepared' N-GQDs using anodic potential 3, 2.5 and $2 \mathrm{~V}$, respectively, and followed by reduction at $-2 \mathrm{~V}$ with average sizes (a) $2.5 \pm 0.3 \mathrm{~nm}$, (b) $4.7 \pm 0.3$ $\mathrm{nm}$ (c) $7.2 \pm 0.3 \mathrm{~nm}$ with their corresponding size distribution histograms and (b) (inset) fringes pattern corresponding to size $4.7 \pm 0.3 \mathrm{~nm}$ showing d-spacing $0.35 \mathrm{~nm}$ and 0.24 . (d) TEM image of N-MWCNTs.

$7.2 \pm 0.3 \mathrm{~nm}$ using a systematic control of the anodic potential at 3, 2.5 and $2 \mathrm{~V}$ (vs. QRE), respectively. These data demonstrate a remarkable correlation between the applied potential and the size; higher the applied potential is, smaller is the resulting N-GQDs, and these smaller N-GQDs could be fractionated further by special post-synthetic treatments like dialysis and density gradient ultracentrifugation to get a narrower dispersion. ${ }^{19,20}$ The potential of oxidation plays an important role in controlling the average size of N-GQDs. More significantly, these N-GQDs show a d-spacing of 0.35 and $0.24 \mathrm{~nm}$ corresponding to the (002) and (1120) crystal phases, respectively, which is in excellent agreement with what is known for N-GQDs prepared by using independent chemical and physical routes (figure $1 \mathrm{~b}$ ) and in agreement with the XRD (figure 3b) ${ }^{19,20}$ Although the exact mechanism of electricfield-assisted breakdown of N-MWCNTs to NGQDs is not very clear, the critical role played by the applied potential in controlling the size of N-GQDs suggests that $\mathrm{N}$ MWCNTs get exfoliated faster due to synergetic effect of applied potential and intercalation of supporting electrolyte ions due to the presence of heteroatom and in the next step (potential $-2 \mathrm{~V}$ for $4 \mathrm{~h}$ ) they presumably get reduced to N-GQDs.

XPS data in the form of BE values are invaluable to discriminating N-MWCNTs from N-GQDs and we have used this also to calculate the extent of different types of nitrogen quantitatively. The XPS of N1s spectra could be deconvoluted (figure 2) into four sub-peaks, which are due to wellknown spin-orbit coupling levels. These four $\mathrm{N}$ sub-peaks are assigned to the pyridinic- $\mathrm{N}$, pyrrolic- $\mathrm{N}$, quaternary- $\mathrm{N}$ and pyridine $\mathrm{N}$-oxide atoms at 397.4, 399.1, 401.3 and $402.8 \mathrm{eV}$, respectively. ${ }^{21}$ The calculated nitrogen content from the XPS data for N-MWCNTs $(2.9 \mathrm{wt} \%)$ shows a close resemblance to that of N-GQDs (2.76 wt \%) with perhaps a higher pyridinic-N content (figure $2 \mathrm{~d}$ ). The nitrogen content, however, is higher in pristine N-MWCNTs, despite a minor decrease, with an increase in the oxidation degree promoted by the amount of oxidation. Indeed, it is known that primary and secondary amines could be degraded by oxidation to aldehydes and ketones, thus loosing nitrogen atoms, concomitant with the generation of ammonia. $^{21,22}$

The Raman technique is a powerful and non-destructive tool for the characterization of GQDs. ${ }^{13}$ The $G$ band is assigned to the $\mathrm{E}_{2 \mathrm{~g}}$ vibrational modes of the aromatic domains, whereas the D band arises from the defective or $\mathrm{sp}^{3}$ carbon of the graphitic domains. Usually, the intensity ratio of 'disorder' $\mathrm{D}$ to crystalline $\mathrm{G}\left(I_{\mathrm{D}} / I_{\mathrm{G}}\right)$ is used to compare the structural order for crystalline and amorphous graphitic systems. Furthermore, a typical Raman spectrum of these materials shows broad peaks at about 1345 and $1590 \mathrm{~cm}^{-1}$, which correspond to the $\mathrm{D}$ and $\mathrm{G}$ bands, respectively (figure $3 \mathrm{a})$. The relative intensity $\left(I_{\mathrm{D}} / I_{\mathrm{G}}\right)$ ratios for N-MWCNTs and N-GQDs are 1.13 and 1.55 , respectively. The full-width at half-maximum of the D band of N-GQDs is, nevertheless, broader compared to that of pristine GQDs, implying more disorder perhaps due to the presence of $\mathrm{N}$ atoms in the carbon framework. ${ }^{27}$ The G-band position in pristine N-MWCNTs appears at $1585 \mathrm{~cm}^{-1}$, and interestingly, after the electrochemical oxidation and followed by reduction the position of $G$ peak is shifted to $1587 \mathrm{~cm}^{-1}$ for N-GQDs.

Also figure $3 \mathrm{~b}$ shows a comparison of the XRD pattern of N-MWCNTs and N-GQDs revealing the broadening of (002) peak at $2 \theta=25.4^{\circ}$ of $\mathrm{N}-\mathrm{GQD}$ due to smaller size. ${ }^{19,20}$ The increase in interlayer distance is attributed to the oxygen-containing groups introduced in the exfoliation and oxidation of N-MWCNTs. The interlayer spacing of NGQDs strongly depends on their oxidation degree, that is, the attached hydroxyl, epoxy/ether, carbonyl and carboxylic acid groups, which distributed on basal plane can increase the interlayer spacing of N-GQDs.

Photoluminescence spectra show a broad excitationdependent emission, perhaps due to the distribution of different emissive sites, and clearly show red shift in the emission at fixed excitation wavelength $(320 \mathrm{~nm})$ for different size $\mathrm{N}$-GQDs (figure 4). ${ }^{19,20} \mathrm{~N}$-GQDs show blue luminescence (as shown in scheme 1) as compared to green luminescent in pristine GQDs, may be due to the presence of nitrogen. This indicates that N-GQDs possess unique topology compared to that of GQDs which is useful for electrocatalysis particularly for oxygen reduction.

A preliminary screening of the electrocatalytic activity of the N-GQDs towards ORR in alkaline medium was carried out by CV. More specifically, nitrogen- and oxygensaturated $0.1 \mathrm{M} \mathrm{KOH}$ aqueous solution was used as the 

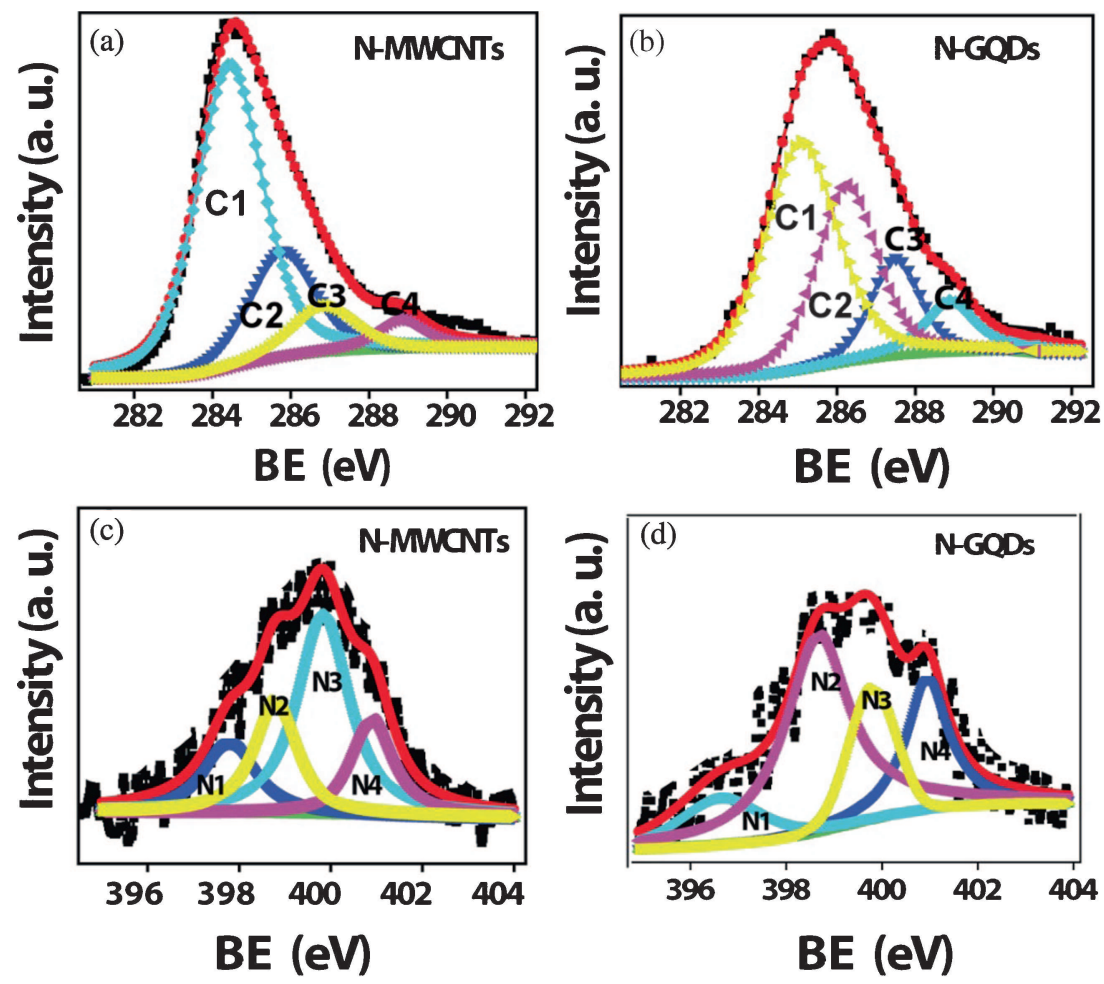

Figure 2. (a) Comparative XPS showing the percentage of carbon, nitrogen and oxygen in N-MWCNTs and N-GQDs, respectively; (b and c) deconvoluted N1s spectra of N-GQDs.
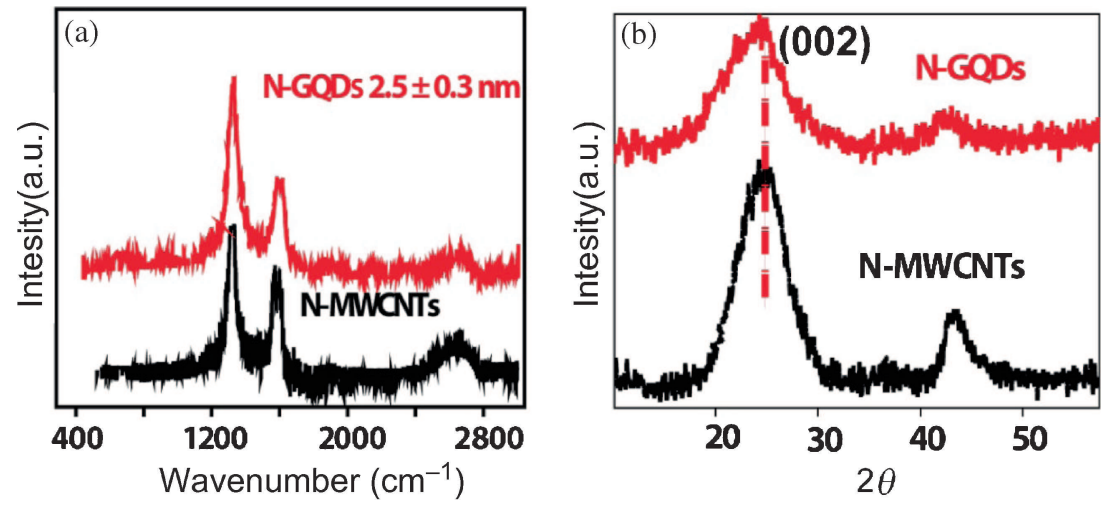

Figure 3. (a) Comparative Raman spectra of N-MWCNTs and N-GQDs. (b) Comparative XRD pattern of N-MWCNTs and N-GQDs.

electrolyte, and current-voltage profiles were measured at a typical scan rate of $50 \mathrm{mV} \mathrm{s}^{-1}$. In all the cases, mass loading was maintained as $20 \mu \mathrm{g}$ on the glassy carbon working electrode (diameter $3 \mathrm{~mm}$ ). Accordingly, figure 5a shows comparative $\mathrm{CV}$ curves of $\mathrm{N}$-GQDs in an $\mathrm{N}_{2}$-saturated 0.1 $\mathrm{M} \mathrm{KOH}$ aqueous solution, exhibiting a pseudo-capacitive behaviour. Moreover, a well-defined reduction peak occurs at around $0.7 \mathrm{~V}$ (vs. NHE) more distinctly in the $\mathrm{O}_{2}$-saturated $0.1 \mathrm{M} \mathrm{KOH}$ aqueous solution, indicating that $\mathrm{O}_{2}$ is more efficiently reduced on the electrode coated with $\mathrm{N}$ -
GQDs. Furthermore, the size-dependent CV (supplementary figure S1) shows an improved pseudo-capacitance as well as a more kinetically facile (higher cathodic peak current density) behaviour with decreasing the size of N-GQDs in $\mathrm{N}_{2}$ and $\mathrm{O}_{2}$-saturated electrolytes, respectively (figure $5 \mathrm{a}$ and supplementary figure S2). The cathodic peak current density scales found in an order of diminishing size of $\mathrm{N}$ GQDs-1 $\left(-0.4 \mathrm{~mA} \mathrm{~cm}^{-2}\right)<\mathrm{N}-$ GQDs-2 $\left(-0.43 \mathrm{~mA} \mathrm{~cm}^{-2}\right)<$ N-GQDs-3 $\left(-0.74 \mathrm{~mA} \mathrm{~cm}^{-2}\right)$ provide an important evidence for size-dependant electrochemical reactivity. As 

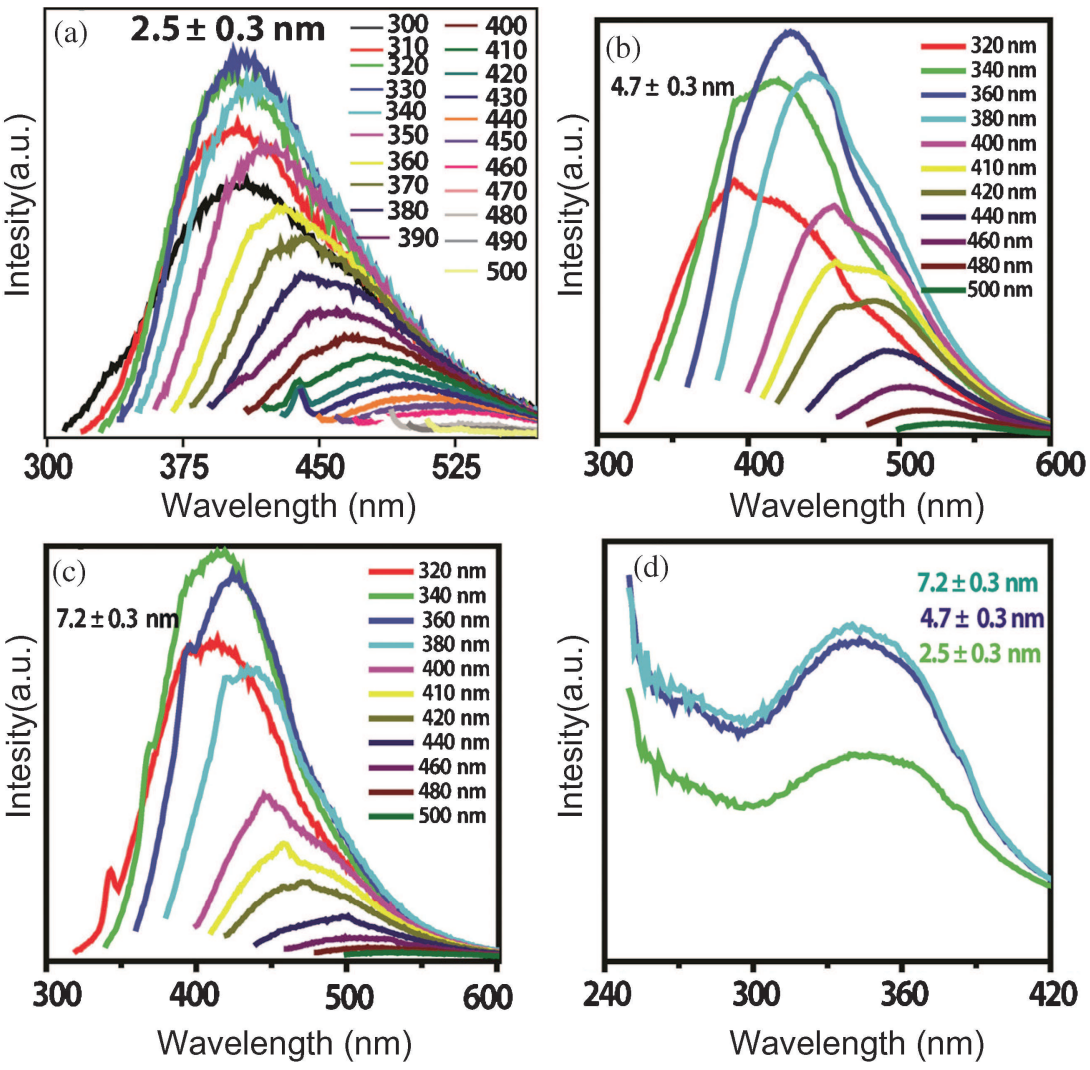

Figure 4. (a-c) Comparative different excitation-dependent emission for various sizes of N-GQDs. (d) Photoluminescence excitation spectra collected at $400 \mathrm{~nm}$.

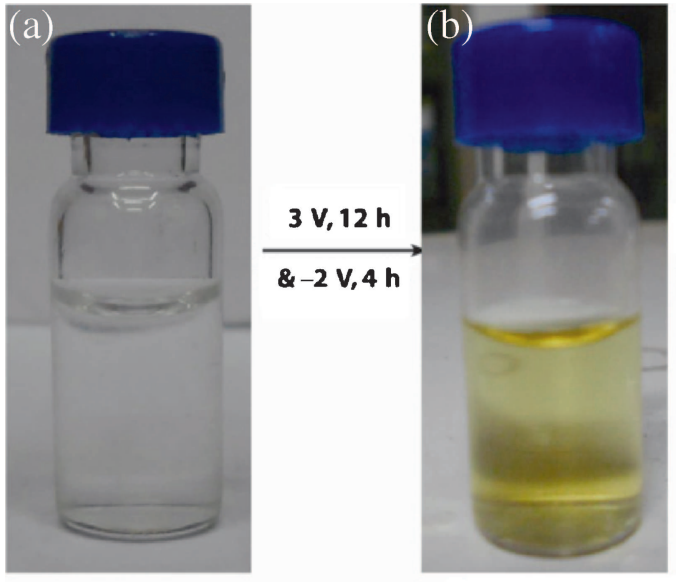

Before

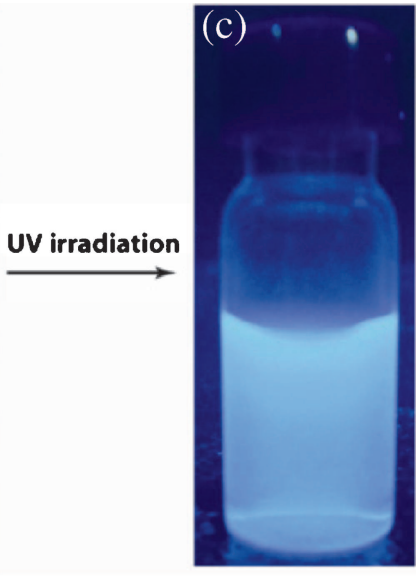

Blue luminescent N-GQDs

Scheme 1. Optical images corresponding to various stages of the synthesis of NGQDs (size $2.5 \pm 0.3 \mathrm{~nm}$ ) from N-MWCNTs using $0.1 \mathrm{M}$ TBAP in acetonitrile at $3 \mathrm{~V}$ for $12 \mathrm{~h}$ followed by their reduction at $-2 \mathrm{~V}$ for $4 \mathrm{~h}$ in the same electrolyte.

the size of GQDs changes, the percentage and exposed $\mathrm{N}$-sites will be more leading to size-dependant reactivity. Second, as the size is smaller the surface-to-volume ratio also increases leading to more number of active defective sites, which are more responsible for the effective dissociative adsorption of oxygen. Hence, the activity and rate constant changes with respect to the size. Linear sweep voltammetry (LSV) experiments were carried out at a scan rate of $10 \mathrm{mV} \mathrm{s}^{-1}$ with the $\mathrm{O}_{2}$-saturated electrolyte in order to estimate the actual kinetics of ORR in 

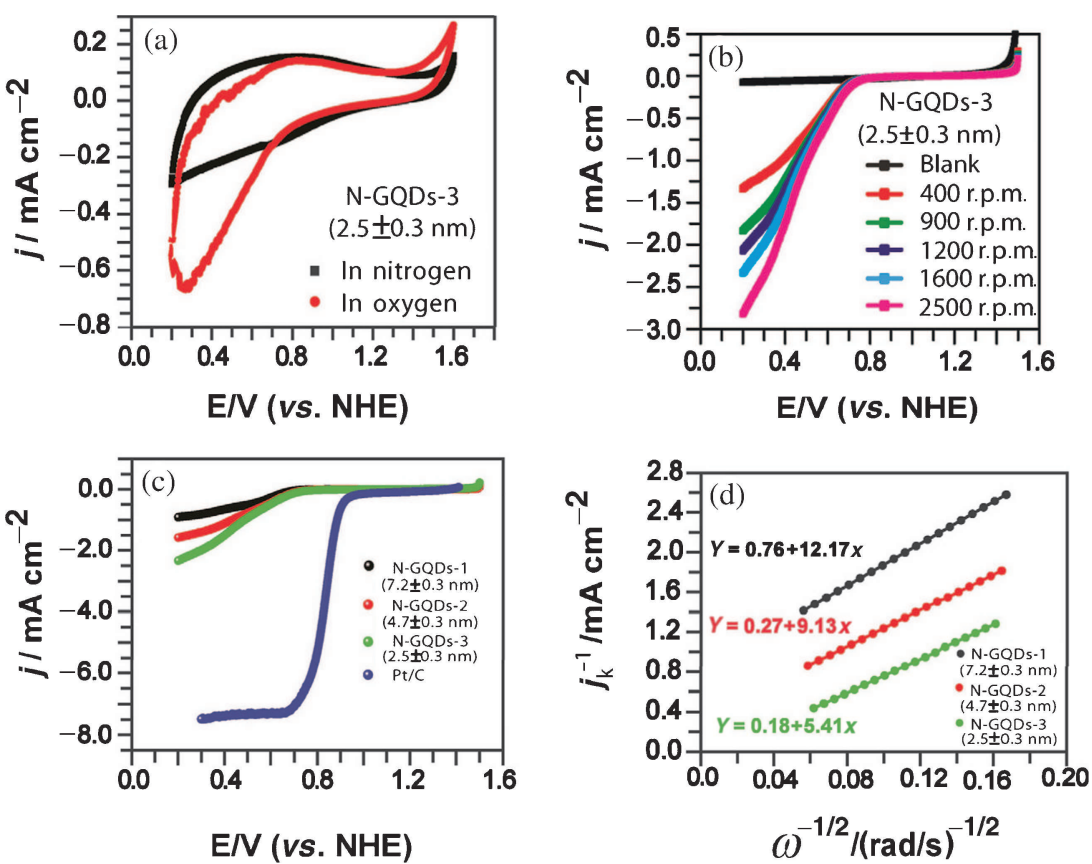

Figure 5. (a) Comparative cyclic voltammograms of N-GQDs-3 in $\mathrm{N}_{2}$ - and $\mathrm{O}_{2}$-saturated $0.1 \mathrm{M} \mathrm{KOH}$ electrolyte at a scan rate of $50 \mathrm{mV} \mathrm{s}^{-1}$, (b) Linear sweep voltammograms (LSV) of N-GQDs-3 in $\mathrm{O}_{2}$-saturated $0.1 \mathrm{M} \mathrm{KOH}$ with different rotation speed of working electrode at a scan rate of $10 \mathrm{mV} \mathrm{s}^{-1}$, (c) comparative LSV of N-GQDs-1, -2 and -3 with that of commercially available Pt/C (20 wt\%), performed at 1600 r.p.m. at scan rate of $10 \mathrm{mV} \mathrm{s}^{-1}$ in $\mathrm{O}_{2}$-saturated $0.1 \mathrm{M} \mathrm{KOH}$ electrolyte and (d) comparative Koutecky-Levich (K-L) plot of N-GQDs-1, -2 and -3 obtained at $0.6 \mathrm{~V}$ from the corresponding LSV data.

a quantitative manner by measuring currents at various rotation speeds of 400, 900, 1200, 1600 and 2500 r.p.m. (figure 5b and supplementary figure S3). Rotation of working electrode will minimize the diffusion-limitation effect. During rotation of working electrode (400-2500 r.p.m.), the potential regions under kinetic and oxygen mass transportlimiting control are noticeably observed and the current density increases with increasing the rotation rate, perhaps due to better enhanced diffusion of electrolyte and mass transfer characteristics. ${ }^{6}$ LSV results indeed confirm that the N-GQDs have size-dependent electrocatalytic activity for ORR, often comparable to that of $\mathrm{Pt} / \mathrm{C}-\mathrm{a}$ benchmark electrocatalyst used for fabricating fuel cells. ${ }^{25}$ Figure $5 \mathrm{c}$ shows a comparison of the LSV curves obtained for N-GQDs 1-3 and commercial $20 \mathrm{wt} \% \mathrm{Pt} / \mathrm{C}$ at 1600 r.p.m. Interestingly, N-GQDs-3 $(0.75 \mathrm{~V})$ show a $30 \mathrm{mV}$ higher (more positive) onset potential shift compared with that of N-GQDs-2 $(0.72 \mathrm{~V})$ and $70 \mathrm{mV}$ than that of N-GQDs-1 (0.68 V). These observations clearly indicate that smaller N-GQDs have higher positive onset potential compared with that of larger size of N-GQDs, attributed to the confinement effect and more exposed $\mathrm{N}$-sites due to smaller size as well as higher surface-to-volume ratio. Moreover, LSV curves does not show saturation currents corresponding to oxygen reduction. ${ }^{28}$ As the Pt-based catalyst has higher dissociative adsorption capacity, mass trasfer controls the rate and consequently, the limiting currents are clearly seen as per the well-known four-electron reduction pathway during oxygen reduction. However, the activity of $\mathrm{N}$-doped carbon structure totally depends on the amount and type of nitrogen present per area in the carbon framework. We have mixed types of nitrogen present in the system, and this along with higher $\mathrm{iR}$ drop (intrinsic as well as due to larger thickness) is responsible for the lack of e saturation currents in the case of GQDs. However, all these N-GQDs have less positive onset potential than that for $\mathrm{Pt} / \mathrm{C}(0.92 \mathrm{~V})$. The more negative onset potential and lower cathodic current density of N-GQDs as compared with $\mathrm{Pt} / \mathrm{C}$, at same mass loading, indicates that N-GQDs have a greater overpotential and its electrocatalytic activities are not as good as commercial Pt/C. However, the N-GQDs had shown to be comparable current with that by $\mathrm{Pt} / \mathrm{C}$, especially, if we consider that the N-GQDs have much greater molecular weight, and thus we may expect lesser active sites ${ }^{23,24}$ in the electrocatalyst even at same mass loadings. Furthermore, Koutecky-Levich (K-L) plots (supplementary figure $\mathrm{S} 4$ ) which is plot of $j_{\mathrm{k}}^{-1} v s . \omega^{-1 / 2}$, have been plotted to calculate the number of electron transfer and actual kinetics of ORR by using following equations:

$$
\begin{aligned}
& \frac{1}{j}=\frac{1}{j_{\mathrm{k}}}+\frac{1}{j_{\mathrm{L}}} \\
& \frac{1}{j}=\frac{1}{B \omega^{1 / 2}}+\frac{1}{j_{\mathrm{k}}}
\end{aligned}
$$




$$
\begin{aligned}
& B=0.62 n \mathrm{~F} A C_{0}^{*} D_{0}^{2 / 3} v^{-1 / 6} \\
& j_{\mathrm{k}}=n \mathrm{~F} A k C_{0}^{*},
\end{aligned}
$$

where $j$ is the measured current density, $j_{\mathrm{L}}$ is the diffusionlimiting current density and $j_{\mathrm{k}}$ is the kinetic current density, $\mathrm{F}$ is the Faraday constant $\left(96486.4 \mathrm{C} \mathrm{mol}^{-1}\right), D_{\mathrm{o}}$ is the diffusion coefficient of oxygen $\left(1.9 \times 10^{-5} \mathrm{~cm}^{2} \mathrm{~s}^{-1}\right), v$ is the kinematic viscosity $\left(0.01 \mathrm{~cm}^{2} \mathrm{~s}^{-1}\right), C_{\mathrm{o}}^{*}$ is the bulk concentration of oxygen and $\omega$ is the angular rotation $(\omega=2 \pi f, f$ is rotation speed), $n$ is number of electron transferred during ORR, and $k$ is the electron transfer rate constant.

Mechanistic studies show that the ORR catalysed by the N-GQDs can occur via a four-electron reduction pathway to produce water directly rather than the two-electron pathway with peroxide as an intermediate. ${ }^{25}$ From the slope of the $\mathrm{K}-\mathrm{L}$ plots (figure $5 \mathrm{~d}$ ) obtained from the LSV data, the number of electrons transferred per oxygen molecule is calculated to be about 3.7 , consistent with a four-electron process. Therefore, similar catalytic mechanisms may be valid to the N-GQDs compared to other N-doped carbon system. ${ }^{19,20}$ Although two-electron pathway to form peroxide also exists, the dominant mechanism of N-doped GQD systems is mainly the four-electron pathway. As shown by the XPS, the percentage of pyridinic- $\mathrm{N}$ is more when compared with the rest. So, we believe that the catalytic activity (nearly equal to four electrons) is mainly the contribution by pyridinic-N, which is well in agreement with the calculated $n$-values from $\mathrm{K}-\mathrm{L}$ plots. The size-dependent catalytic activity of the N-GQDs for ORR has important implications in understanding and improving the catalytic activities of $\mathrm{N}$-doped carbon materials, especially for fuel cell/water electrolyser applications. However, the yield of N-GQDs is limited due to smaller surface area of working electrode, and bulk production is quite possible for fuel cell application using large area working electrode.

\section{Conclusions}

A unique electrochemical transformation of N-MWCNTs to N-GQDs has been demonstrated and the resultant $\mathrm{N}$ GQDs display many unusual size-dependant optoelectronic and electrocatalytic properties. The presence of nitrogen dopants in the carbon framework not only causes faster unzipping of N-MWCNTs, but also provides more low activation energy site for enhancing the electrocatalytic activity for ORR. These results on the size-dependent electrocatalytic activity of N-GQDs for ORR, as exemplified by the smaller-sized N-GQDs $(2.5 \pm 0.3 \mathrm{~nm})$, may be a promising alternative to $\mathrm{Pt}$-based electrocatalysts for fuel cell applications.

\section{Acknowledgement}

This study was financially supported by the Council of Scientific and Industrial Research (CSIR), India. D B Shinde and
V M Dhavale are grateful to Dr Saurav Pal, Director of National Chemical Laboratory, Pune, Maharashtra, India, for providing infra-structure and constant support. D B S also thanks to Mr Pankaj and Miss Shravani for TEM images.

\section{Electronic Supplementary Material}

Supplementary Material pertaining to this article is available on the Bulletin of Materials Science website (www.ias.ac.in/ matersci).

\section{References}

1. Sheng W, Gasteiger H A and Shao-Horn Y 2010 J. Electrochem. Soc. 157 B1529

2. Norskov J K, Rossmeisl J, Logadottir A, Lindqvist L, Kitchin J R, Bligaard T and Jónsson H 2004 J. Phys. Chem. B 108 17886

3. Dhavale V M, Unni S M, Kagalwala H N, Pillai V K and Kurungot S 2011 Chem. Commun. 473951

4. Palaniselvam T, Kannan R and Kurungot S 2011 Chem. Commun. 472910

5. Gasteiger H, Kocha S, Sompalli B and Wagner F 2005 Appl. Catal. B $\mathbf{5 6} 9$

6. Gong K, Du F, Xia Z, Durstock M and Dai L 2009 Science 323 760

7. Kundu S, Nagaiah T C, Xia W, Wang Y, Dommele S V, Bitter J H, Santa M, Grundmeier G, Kim H, Lee K, Woo S I and Jung Y 2011 Phys. Chem. Chem. Phys. 1317505

8. Qu L, Liu Y, Baek J B and Dai L 2010 ACS Nano. 41321

9. Panchakarla L S, Subrahmanyam K S, Saha S K, Govindaraj A, Krishnamurthy H R, Waghmare U V and Rao C N R 2009 Adv. Mater. 214726

10. Zhang Z, Zhang J, Chen N and Qu L 2012 Energy Environ. Sci. 58869

11. Dai J Y, Yuan J M and Giannozzi P 2009 Appl. Phys. Lett. 95 232105

12. Meyer J C, Kurasch S, Park H J, Skakalov V, Kunzel D, Grob A, Chuvilin A, Algara-Siller G, Roth S, Iwasaki T, Starke U, Smet J H and Kaiser U 2010 Nat. Mater. 10209

13. Liu H T, Liu Y Q and Zhu D B 2011 J. Mater. Chem. 213335

14. Ma L, Hu H, Zhu L Y and Wang J H 2011 J. Phys. Chem. C 1156195

15. Li Q, Zhang S, Dai L and Li L 2012 J. Am. Chem. Soc. 134 18932

16. Shinde D B and Pillai V K 2012 Chem. Eur. J. 3912522

17. Kim H, Lee K, Woo S I and Jung Y 2011 Phys. Chem. Chem. Phys. 1317505

18. Shinde D B and Pillai V K 2013 Angew. Chem. 522482

19. Zhang M, Bai L, Shang W, Xie W, Ma H, Fu Y, Fang D, Sun H, Fan L, Han M, Liu C and Yang S 2012 J. Mater. Chem. 22 7461

20. Moulder J F 1995 Handbook of X-ray photoelectron spectroscopy. A reference book of standard spectra for identification 
and interpretation of xpsdata, Eden Prairie, MN: Physical Electronics

21. Rawalay S S and Shechter H J 1967 J. Org. Chem. 32 3129

22. Lin Y C and Chiu P W 2010 Appl. Phys. Lett. 9633110

23. Yu S, Zheng W, Wang C and Jiang Q 2010 ACS Nano. 4 7619

24. Zhuo S, Shao M and Lee S T, 2012 ACS Nano. 61059
25. Bron M, Schuhmann W and Muhler M 2009 J. Phys. Chem. C 11314302

26. Li Y, Zhao Y, Cheng H, Hu Y, Shi G, Dai L and Qu L $2011 \mathrm{~J}$. Am. Chem. Soc. 13415

27. Liu R L, Wu D Q, Feng X L and Mullen K 2010 Angew. Chem. 492565

28. Li X, Zhang L, Yoon Y, Weber P K, Wang H, Guo J and Dai H 2009 Science $\mathbf{3 2 4} 768$ 